\title{
Evolutionary research on the expansin protein family during the plant transition to land provides new insights into the development of Tartary buckwheat fruit
}

Wenjun Sun ${ }^{1 \dagger}$, Haomiao $\mathrm{Yu}^{1+}$, Moyang Liu ${ }^{1,2+}$, Zhaotang Ma ${ }^{3}$ and Hui Chen ${ }^{1 *}$ (D)

\begin{abstract}
Background: Plant transitions to land require robust cell walls for regulatory adaptations and to resist changing environments. Cell walls provide essential plasticity for plant cell division and defense, which are often conferred by the expansin superfamily with cell wall-loosening functions. However, the evolutionary mechanisms of expansin during plant terrestrialization are unclear.

Results: Here, we identified 323 expansin proteins in 12 genomes from algae to angiosperms. Phylogenetic evolutionary, structural, motif gain and loss and Ka/Ks analyses indicated that highly conserved expansin proteins were already present in algae and expanded and purified after plant terrestrialization. We found that the expansion of the FtEXPA subfamily was caused by duplication events and that the functions of certain duplicated genes may have differentiated. More importantly, we generated space-time expression profiles and finally identified five differentially expressed FtEXPs in both large and small fruit Tartary buckwheat that may regulate fruit size by responding to indoleacetic acid.

Conclusions: A total of 323 expansin proteins from 12 representative plants were identified in our study during terrestrialization, and the expansin family that originated from algae expanded rapidly after the plants landed. The EXPA subfamily has more members and conservative evolution in angiosperms. FtEXPA1, FtEXPA11, FtEXPA12, FtEXPA19 and FtEXPA24 can respond to indole-3-acetic acid (IAA) signals and regulate fruit development. Our study provides a blueprint for improving the agronomic traits of Tartary buckwheat and a reference for defining the evolutionary history of the expansin family during plant transitions to land.
\end{abstract}

Keywords: Expansin, Terrestrialization, Phylogenetic, Evolutionary research, Tartary buckwheat

\footnotetext{
* Correspondence: chenhui@sicau.edu.cn

${ }^{\dagger}$ Wenjun Sun, Haomiao Yu and Moyang Liu contributed equally to this work. 'College of Life Science, Sichuan Agricultural University, Ya'an 625014, China

Full list of author information is available at the end of the article
}

(c) The Author(s). 2021 Open Access This article is licensed under a Creative Commons Attribution 4.0 International License, which permits use, sharing, adaptation, distribution and reproduction in any medium or format, as long as you give appropriate credit to the original author(s) and the source, provide a link to the Creative Commons licence, and indicate if changes were made. The images or other third party material in this article are included in the article's Creative Commons licence, unless indicated otherwise in a credit line to the material. If material is not included in the article's Creative Commons licence and your intended use is not permitted by statutory regulation or exceeds the permitted use, you will need to obtain permission directly from the copyright holder. To view a copy of this licence, visit http://creativecommons.org/licenses/by/4.0/. The Creative Commons Public Domain Dedication waiver (http://creativecommons.org/publicdomain/zero/1.0/) applies to the data made available in this article, unless otherwise stated in a credit line to the data. 


\section{Background}

Land plant radiation and colonization are important keystones in the evolutionary history of living organisms, which have created the ecological diversity on Earth that we see today. This transition was accompanied by complex and long biological evolution, which included morphological, physiological, and genetic changes, to cope with the terrestrial environment and its challenging conditions [1,2]. The cell wall plays a key role in plant growth and development, material transport, pathogen resistance, cell division and differentiation, organ senescence and shedding. It also provides the necessary mechanical support for plant cells and the plasticity that is necessary for protection against external intrusion $[3,4]$. The number and volume of plant cells always change dynamically, and both are regulated by cell wall plasticity during plant growth [5]. Studies have shown that the role of expansin proteins in the cell wall is critical to achieve this necessary plasticity [5]. Expansin is an important plant growth-regulating divisor that can realize the continuous assembly, remodeling and decomposition of cell walls [6]. It has significant functionality in many stages of plant growth and development [7], such as stem growth and internode elongation [8], fruit ripening [9], seed germination [10], control of flowering time and flower size [11], root growth [12] and leaf development [13].

Expansin proteins contain 250-275 amino acid residues [14] and consist of two conserved domains. The Nterminal conserved domain I (DPBB), which contains approximately $120-135$ amino acids, is homologous to glycoside hydrolase family-45 (GH45). Previous studies have shown that there is no $\beta$-glucan sugar hydrolysis at the $\mathrm{N}$-terminus of expansin proteins [15]. Another domain (domain II in the C-terminus) contains approximately 90-120 amino acids and has higher similarity with Group-II pollen allergen proteins (G2A family) and presumably is a polysaccharide binding domain (PLN) based on the polar residues on the surfaces of proteins and conserved aromatics [16]. To date, no other proteins containing domain II congeners have been found except for the G2A families [17]. A recent study established a 3D model of the FaEXPA2 protein that was involved in strawberry fruit softening and determined that FaEXPA2 formed a more stable complex with cellulose than other ligands via the different residues present in the open groove surface of its two domains [18]. Similarly, molecular dynamics showed that the FaEXPA5 protein is involved in strawberry fruit softening and can interact with ligands through the residues present in the open groove along the two domains [19]. Expansin proteins are cocoded by multiple gene families and are divided into the $\alpha$-expansin (EXPA), $\beta$-expansin (EXPB), expansin-like A (EXLA), and expansin-like B (EXLB) subfamilies according to their phylogeny [20]. While
EXLA and EXLB also possess two typical expansin protein domains, there is no experimental evidence that they also have the function of loosening cell walls [21]. Generally, EXPA is widely found in dicotyledonous and monocotyledonous plants, except non-Poaceae, while EXPB is mainly found in monocotyledonous plants [15]. Expansin proteins have been studied in many important species, including Arabidopsis thaliana (A. thaliana) [22], tea [23], Solanum lycopersicum [24], Z. mays [25], Glycine max [26], cotton [27] and wheat [28]. The EXPA subfamily was the first subfamily to be identified that contains cell wall-loosening proteins, which can quickly induce relaxation of the cell wall without lytic activity [29]. AtEXPA7, which is an EXPA family gene that is specifically expressed in root hair cells, was isolated from A. thaliana, and its biological function was detected by using RNA interference. The results showed that AtEXPA7 played an indispensable role in root hair tip growth [30]. Overexpression of AtEXPA2 promotes seed germination, while inhibition of its expression leads to a delay in seed germination [31]. Meanwhile, studies have shown that AtEXPA2 may regulate seed germination through the GA signaling pathway [31]. The EXPB subfamily consists of two subgroups. Group- 1 proteins are highly expressed in grass pollen [32] and can relax cell walls without destroying them [32]. Research on EXPA and EXPB is relatively deeper [33]. Recent reports have also confirmed the role of expansin proteins in fruit enlargement $[34,35]$, which provides new insights for improving crop agronomic traits.

Current agricultural studies are centered on the main staple crops, including rice, wheat and maize. However, this narrow research scope is not promising for providing systematic solutions to the challenges of food security and poverty [36]. Adding nutrient-rich pseudocereals to major cereals is a potential strategy to improve dietary diversity and provide alternative food stocks. Tartary buckwheat (Fagopyrum tataricum) is a versatile pseudocereal that is known as the golden crop [36]. It is also a traditional Chinese grain crop that is widely cultivated in China. Because of its strong environmental adaptability, it has become the main food source for people living in severe environments such as the southwest plateau of China [37]. Tartary buckwheat fruits are rich in starch, proteins, dietary fiber, vitamins and other nutrients [38]. In addition, the flavonoid contents in Tartary buckwheat are significantly higher than those of other foods, and proper intake can help organisms due to their antioxidant and anti-aging properties, as well as their ability to lower blood pressure and reduce the risk of arteriosclerosis [39]. Because of its important value in food and medicine, Tartary buckwheat has received more attention from breeding and genetic researchers in recent years. Some challenges in the breeding of Tartary 
buckwheat, such as increasing the dehulling efficiency of fruit, improving fruit quality, and increasing fruit size, remain to be solved [40].

Considering the important role of expansin proteins in plant development and adaptation to complex terrestrial environments, we identified 323 expansin proteins in 12 genomes from algae to angiosperms. We studied these proteins by performing phylogenetic analysis, gene structure and motif composition analysis, cis-acting element identification of promoter regions, and gene duplication. We also analyzed the origin and evolution of expansin proteins in representative plants during plant terrestrialization. More importantly, we identified five candidate genes from the EXPA subfamily that may improve the important agronomic traits of Tartary buckwheat, which was accomplished by combining the expression of 37 genes in different tissues and organs, especially in the important stages of fruit development. In summary, our study identified the FtEXP gene family for the first time. The conservation and evolution of this species in the process of plant landing are discussed, and its potential regulatory roles in fruit development and hormone response are determined, which provides new insights for Tartary buckwheat breeding.

\section{Results}

Global identification and evolution of Expansin proteins from algae to land plants

To further understand the evolutionary history of expansin during plant transitions to land, we identified 323 expansin genes by using BLAST and profile HMM searches of two algae (Chlamydomonas reinhardtii and Volvox carteri); three bryophytes (Marchantia polymorpha, Physcomitrella patens and Sphagnum palustre); early angiosperms (Amborella trichopoda); two monocotyledons (Oryza sativa and Zea mays) and four dicotyledons (F. tataricum, Arabidopsis, Vitis vinifera and Coffea arabica) (Fig. 1, Table S1). We divided the expansin family into four subfamilies (EXPA, EXPB, EXLA and EXLB) according to the distribution and structural characteristics of the Arabidopsis EXP (AtEXP) members [20] (Fig. 1, Table S2).

Furthermore, the numbers of expansins in each subgroup of these species were investigated (Fig. 1, Table S1). There were fewer members of the algae EXPA subfamily and more members of the EXLB subfamily, which was in sharp contrast to higher plants (Fig. 1). Interestingly, up to 32 members of the EXPA subfamily were found in $M$. polymorpha, while other subfamily members were not

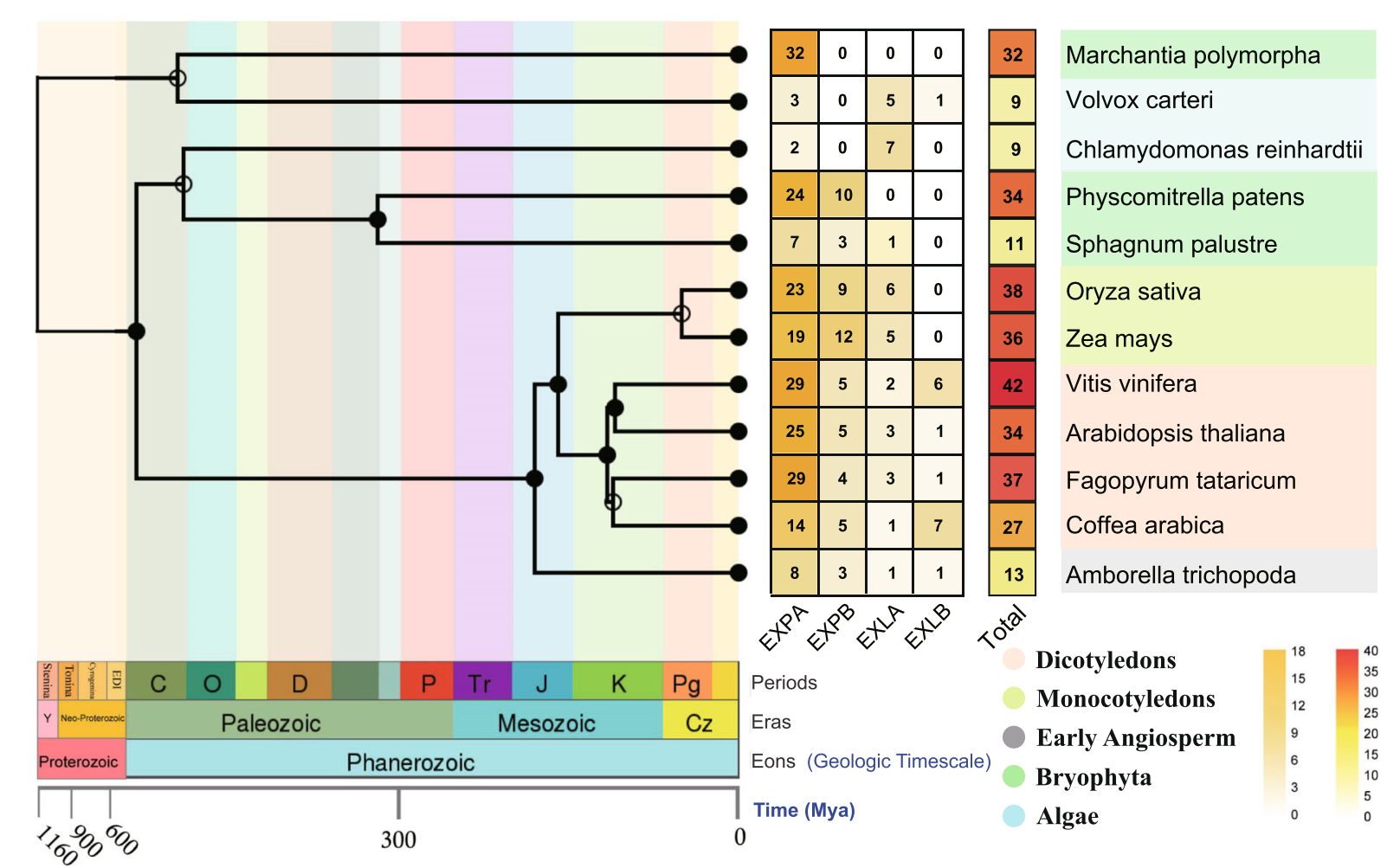

Fig. 1 Phylogeny and diversity of expansin proteins in 12 species. A species tree was constructed using the online software TIMETREE (http:// www.timetree.org/). The number of members in different subfamilies is expressed by a color scale. The blue, green, gray, light green and orange colors represent algae, Bryophyta, early angiosperms, monocotyledons and dicotyledons, respectively 
found, which shows that the EXPA subfamily began to expand as the plant made the transition to land. In monocotyledon species, EXPB was the larger subfamily, while EXPA was the larger subfamily in dicotyledons. EXLB was present only in early angiosperms and dicotyledons but not in other plants except for $V$. carteri, and EXPA arose early in the evolution of bryophytes and was conserved across land plants (Fig. 1).

\section{Analysis of phylogeny and evolution suggests that the FtEXPA subfamily has rich members and special structures}

We identified 37 expansin proteins in the Tartary buckwheat genome and assembled the basic information for these genes, such as Mw, PI, subcellular localization, CDS and protein sequence (Table S3-4). Based on the multiple sequence alignment of 37 FtEXP proteins and 34 A. thaliana expansin proteins, we reconstructed a maximum likelihood phylogenetic tree to explore the evolutionary relationships of expansin proteins in Tartary buckwheat (Fig. 2). The number of genes in different subfamilies varies. The EXLB subfamily has the lowest number of members (only one gene), and the EXPA subfamily has the largest number of genes (Fig. 2). The number of expansin proteins in each subfamily of Tartary buckwheat is very close to that in A. thaliana.

Furthermore, we mapped all FtEXPs to 8 chromosomes, based on physical location information from the Tartary buckwheat genome generic feature format (Gff) data (Fig. 3). The 37 FtEXPs are unevenly distributed on 8 chromosomes. Most genes are on chromosome 3 (eleven genes), and the fewest are on chromosome 6 (only one gene). The genes on chromosome 7 and chromosome 8 are also less distributed, but each chromosome has a tandem duplicate region. Multiple FtEXPs are distributed on chromosomes 1, 3 and 4, but only one pair of tandemly duplicated genes was detected on chromosome 3 (Fig. 3). Two pairs of EXPA subfamily genes (FtPinG0001244700.01-FtPinG0001244900.01 and FtPinG0009206900.01- FtPinG0009207100.01) from chromosomes 3 and 8 are tandem duplications, which may have contributed to the expansion of the EXPA subfamily to some extent. In addition, 37 FtEXPs were renamed according to their subfamilies and chromosomal distributions (Table S3).

We also investigated the exon-intron organizations of all identified FtEXPs for a deeper understanding of the evolution of this family in Tartary buckwheat (Fig. 4a). Among $37 \mathrm{FtEXPs}$, the number of introns ranged from 0 to 3, and most members of the EXPA subfamily contained 2 introns. Notably, the structure of several members of the EXPA subfamily is special; for example, only FtEXPA6 (FtPinG0002998000.01) contains a PLN domain, and FtEXPA26 (FtPinG0007038600.01) contains five introns, while its exon length is significantly different from those of the other genes (Fig. 4a). Analysis of the motifs was performed through the online MEME software to further study the characteristic regions of the FtEXP proteins (Fig. 4b). Most members of the EXPA subfamily contain motifs 1 to 8 , while most members of the other subfamilies contain motifs $3,4,7,9$ and 10 (Fig. 4b). Notably, some genes contain very few motifs; for example, FtEXPA26 (FtPinG0007038600.01) contains only motif 5, while FtEXPA9 (FtPinG0000802100.01) contains only motifs 3 and 4 . Overall, most genes from the same subfamily have similar motif compositions, and the expansin proteins of the other 11 plants also have conserved domains and general characteristics (Fig. S1-S2, Table S5).

Environmental stress can profoundly affect the growth and development of plants [41]. We analyzed the cisacting elements of 37 FtEXP promoter regions by using PlantCARE software to investigate their responses to the environment. Three environmentally responsive elements were detected, including light-, low temperatureand defense stress resistance-responsive elements, and they were widespread in 37 FtEXPs (Fig. S3). Most hormone-responsive elements (MeJA, auxin, abscisic acid and gibberellin) were also widely distributed in all FtEXPs, except the salicylic acid-responsive elements (Fig. S3). Salicylic acid-responsive elements exist only in the EXPA subfamily, and such responsive elements that are related to plant disease resistance [42] and drought tolerance [43] have attracted our attention.

\section{Gene duplication and evolutionary analysis of Expansin gene families in representative species}

Gene duplication that arises from tandem duplication or during polyploidization and segmental duplication associated with replication is a major factor causing family expansion. For a deeper understanding of the evolution of expansin homologous copy genes, we conducted a syntenic analysis of the expansin proteins from four dicotyledons (F. tataricum, Arabidopsis, C. arabica and $V$. vinifera) and two monocotyledonous plants (O. sativa and $Z$. mays). We detected 14 pairs of segmental duplications on different chromosomes of Tartary buckwheat (Fig. 5a). Most segmental duplication genes also came from the EXPA subfamily (FtPinG0000209500.01, FtPinG0002998000.01, FtPinG0000802100.01, FtPinG0 006622100.01 and FtPinG0004679600.01), which could be another important reason why the EXPA subfamily expanded within species. The results also showed that different pairs of segmental duplication EXP gene pairs were found in the genomes of Arabidopsis (22 pairs), $V$. vinifera (6 pairs), and O. sativa (6 pairs) (Fig. $5 \mathrm{~b}-\mathrm{d}$ ). To explore the different selective constraints of the duplicated FtEXP pairs, we calculated the Ks values and $\mathrm{Ka} /$ 


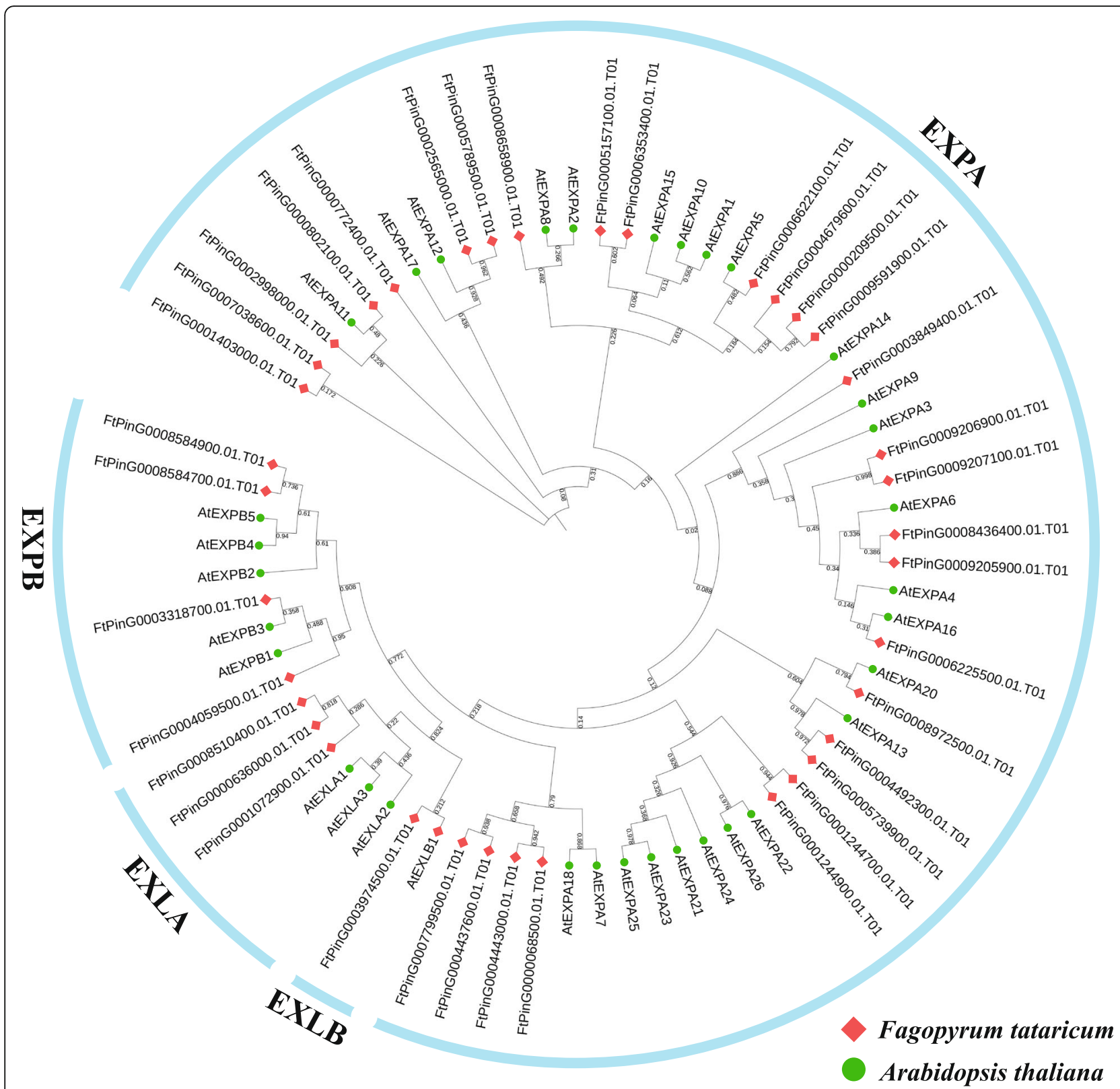

Fig. 2 Phylogenetic tree that represents the relationships among 37 expansin genes of Tartary buckwheat and 34 expansin genes of $A$. thaliana. The phylogenetic tree of the expansin protein sequences of Tartary buckwheat and A. thaliana was constructed with Mega 7.0 by the maximum likelihood method and was visualized by the online tool Interactive Tree Of Life (iTOL) (http://itol2.embl.de/). The genes in Tartary buckwheat are marked in red diamond, while those in A. thaliana are marked in green circle

Ks ratios of each homologous gene pair between Tartary buckwheat and other terrestrial plants (Table S6). The $\mathrm{Ka} / \mathrm{Ks}$ values of the majority of expansin homologous gene pairs were less than 1, especially for the EXPA subfamily, which indicated that expansin genes are highly conserved in evolution and can be important for plant growth and development (Fig. 5e, Table S6).

Previous reports have shown that synteny occurs not only within species; synteny genes between species are often another channel for the rapid evolution of gene families and are prone to copy genes with similar functions [44]. Therefore, we further investigated syntenic genes that are homologous to Tartary buckwheat expansins in representative plants. Syntenic expansin gene pairs are widely found among Tartary buckwheat and Arabidopsis (32 homologous gene pairs), C. arabica (32 homologous gene pairs), $V$. vinifera (15 homologous gene pairs), O. sativa (5 


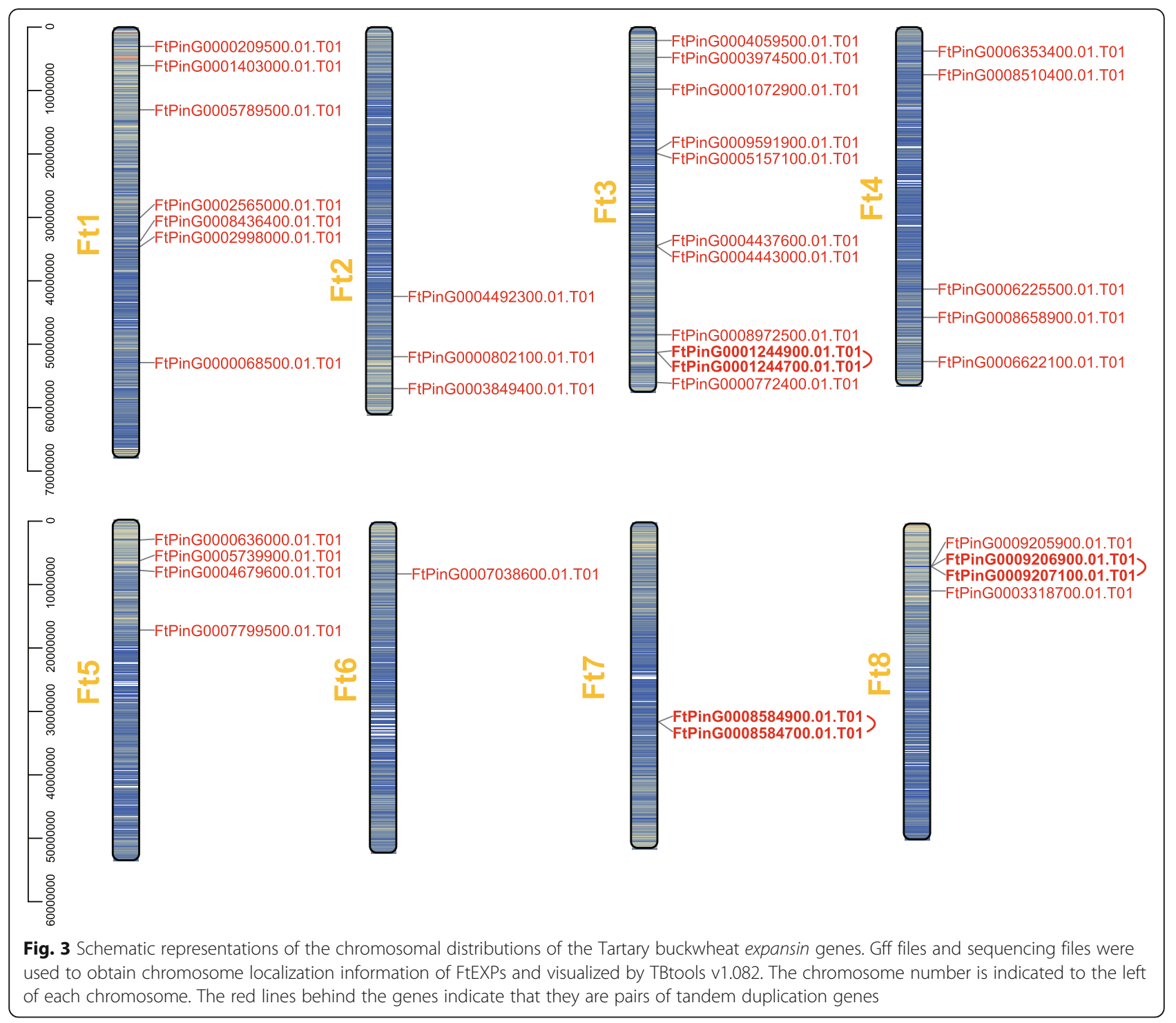

homologous gene pairs), and $Z$. mays (only 1 homologous gene pair) (Fig. 6, Table S7).

\section{Differential expression of EXPA subfamily genes in different tissues of Tartary buckwheat}

Many reports have shown that expansin proteins are closely related to plant growth and development, especially the fruit development of angiosperms; examples include $A$. thaliana [45], wheat [46], rice [47], tomatoes [48], and tobacco [49]. Therefore, we detected the expression of 37 FtEXPs in different tissues of Tartary buckwheat by quantitative real-time polymerase chain reaction (qRT-PCR).

The histograms show that all FtEXPs were expressed except FtPinG0001244700.01. Twenty genes exhibited expression in each tissue. There were some tissue-specific genes, of which FtPinG0000772400.01 was a specific gene that was expressed only in roots, and FtPinG0008584900.01 and
FtPinG0001244900.01 were specific genes that were expressed only in flowers (Fig. 7a). Among the 36 genes, 12 genes had the highest expression levels in roots, and 5 genes had the highest expression levels in stems. Interestingly, we found six FtEXPs with special expression in fruit, including five genes (FtPinG0002998000.01, FtPinG0007038600.01, FtPinG0005157100.01, FtPinG0006353400.01 and FtPinG0006225500.01) with significantly higher expression than in other tissues, and one gene (FtPinG0000802100.01) that was expressed only in fruit. The six special genes were all from the EXLA subfamily, although the FtPinG0000802100.01 expression was relatively low. Members of the EXPA subfamily are generally involved in the regulation of plant fruit development, which has been fully confirmed in previous studies [50].

Moreover, we also provided the correlations among the expression levels of each gene. We can see from the 


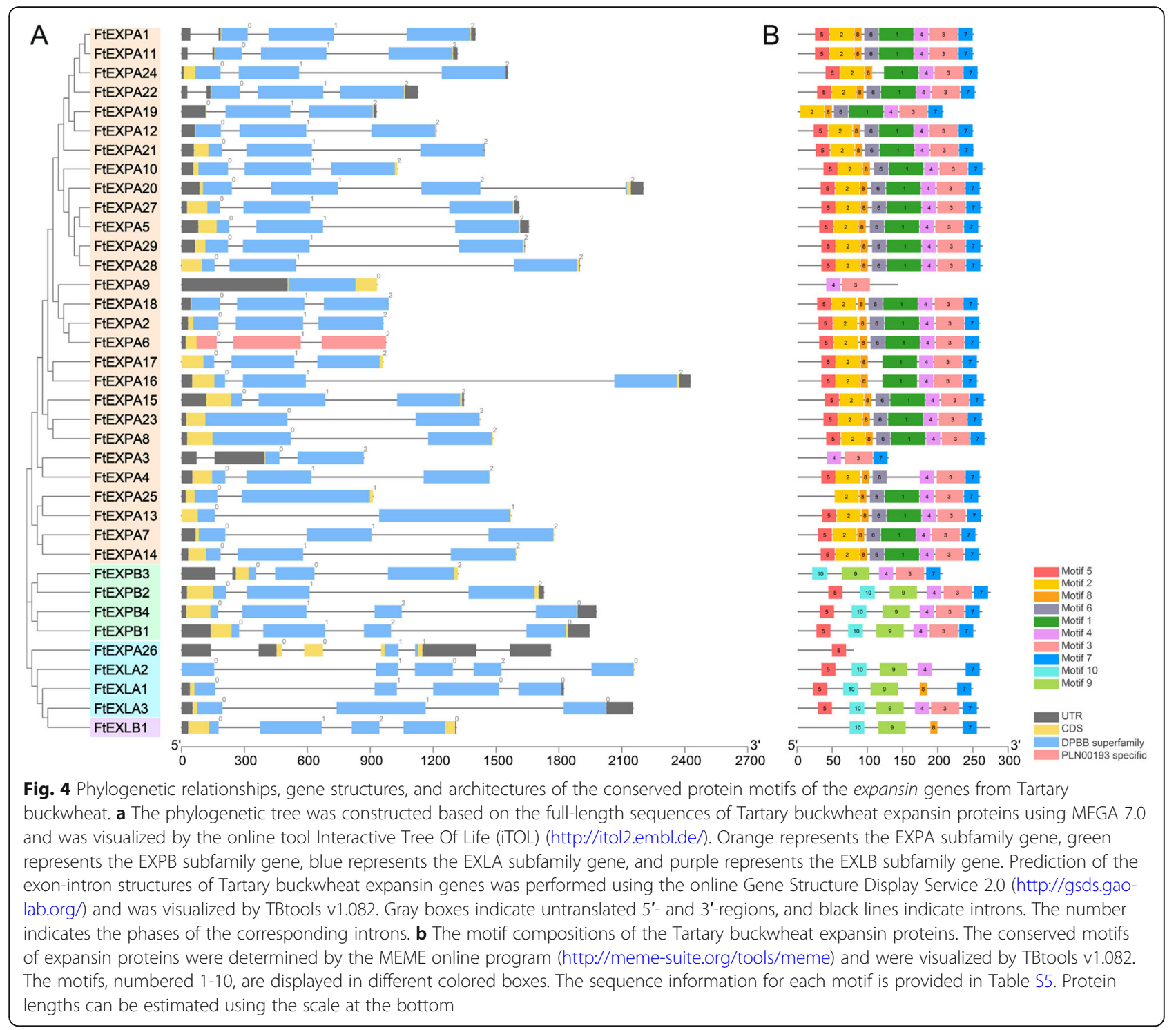

correlation analysis of the 36 genes expressed in different tissues that there were positive correlations among the expression profiles of most genes, especially the six fruit-specific genes mentioned earlier, all of which were significantly positively correlated (Fig. 7b).

\section{Expression patterns of EXPA subfamily members were different in the three important periods of fruit development}

In the preliminary study, we divided Tartary buckwheat into five stages from anthesis to maturation according to embryonic development morphology, among which the green fruit stage (13 DAP), discoloration stage (19 DAP) and initial maturity stage (25 DAP) were the three most important developmental stages [51]. To screen the potential FtEXPs regulating fruit development, we determined the expression of 31 FtEXPs during the three most important fruit development stages (13 DAP, 19 DAP and 25 DAP) by qRT-PCR. The results showed that the expression of 4 genes increased gradually at 13 DAP, 19 DAP and 25 DAP, including three genes from the EXPA subfamily (FtPinG0002998000.01, FtPinG0007 038600.01 and FtPinG0005157100.01) and one gene from EXPB (FtPinG0008584700.01), which was not expressed at 25 DAP. In addition, among the genes that were expressed in all three periods, six genes experienced both upregulation and downregulation. Three EXPA subfamily genes that were specifically expressed in fruit (FtPinG0006353400.01, FtPinG0006255000.01 and FtPinG0000802100.01) were also within the range (Fig. 8a).

From the correlation study of 31 FtEXP expression levels in fruits at different developmental stages, it can be seen that some genes showed significant negative 


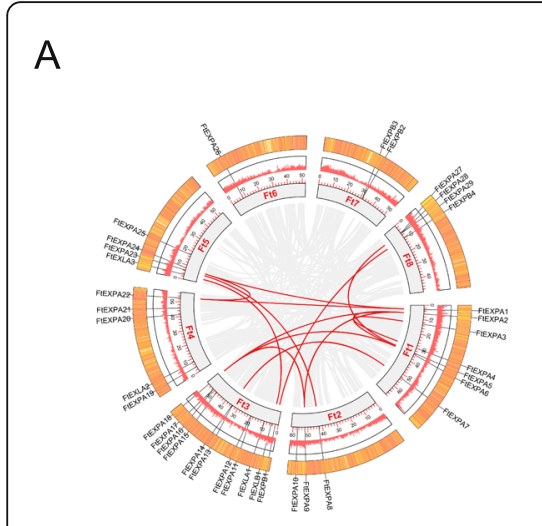

Fagopyrum tataricum

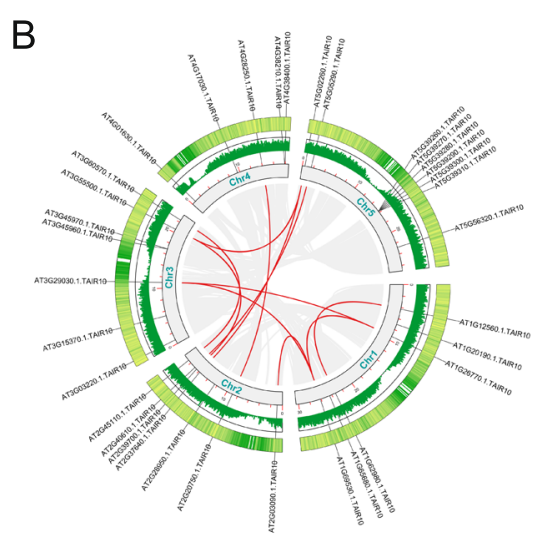

Arabidopsis thaliana

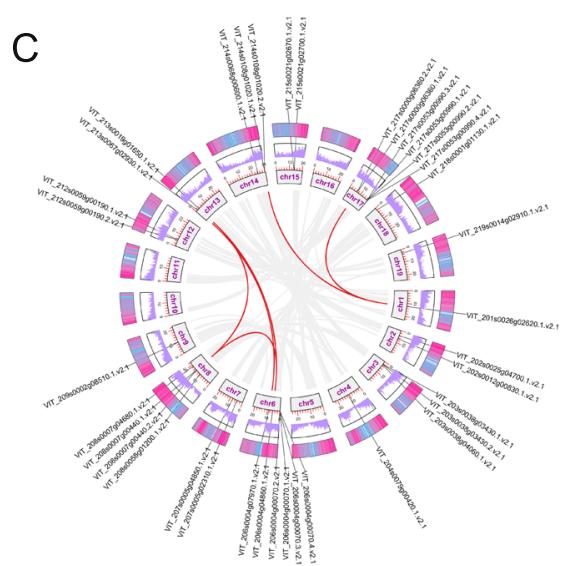

Vitis vinifera

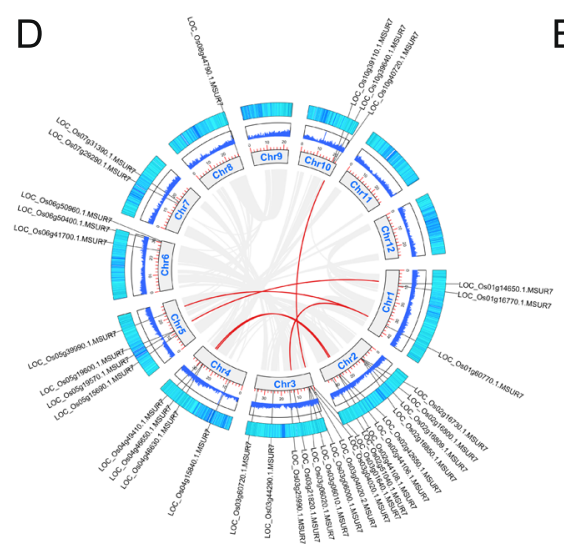

E

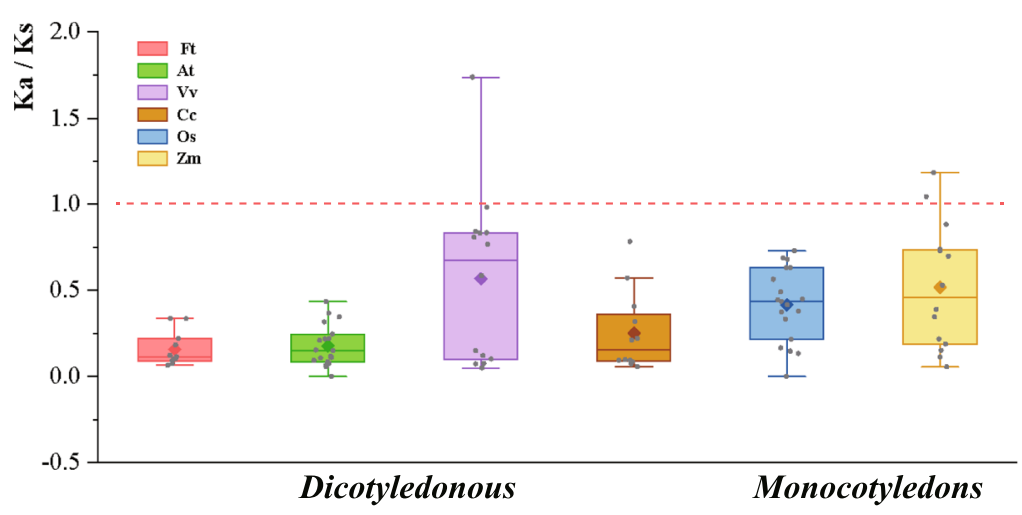

Fig. 5 Schematic representations of the interchromosomal relationships of the expansin genes from different plants. a Analysis of the interchromosomal relationships of the expansin genes from Tartary buckwheat was conducted by using multiple collinear scanning toolkits (MCScanX) and was visualized by TBtools v1.082. Gray lines in the background indicate collinear blocks within the Tartary buckwheat genome, while red lines highlight syntenic expansin gene pairs. $\mathbf{b}$ Analysis of the interchromosomal relationships of the expansin genes from $A$. thaliana was conducted by using multiple collinear scanning toolkits (MCScanX), and was visualized by TBtools v1.082. Gray lines in the background indicate collinear blocks within the A. thaliana genome, while red lines highlight syntenic expansin gene pairs. c Analysis of the interchromosomal relationships of the expansin genes from Vitis vinifera was conducted by using multiple collinear scanning toolkits (MCScanX), and was visualized by TBtools v1.082. Gray lines in the background indicate collinear blocks within the Vitis vinifera genome, while red lines highlight syntenic expansin gene pairs. $\mathbf{d}$ Analysis of the interchromosomal relationships of the expansin genes from Oryza sativa was conducted by using multiple collinear scanning toolkits (MCScanX), and was visualized by TBtools v1.082. Gray lines in the background indicate collinear blocks within the Oryza sativa genome, while red lines highlight syntenic expansin gene pairs. e KaKs_Calculator 2.0 was used to calculate the synonymous (Ks) and nonsynonymous (Ka) substitutions of each homologous expansin gene pair and their ratios (Ka/Ks). The results were visualized using TBtools v1.082

correlations with nearly all genes, such as FtPinG0002998000.01, FtPinG0008510400.01, FtPinG00 07038600.01, FtPinG0006255000.01 and FtPinGO 005157100.01 (Fig. 8b). High gene expression levels in a certain tissue or development phase indicate that the gene may perform certain actions during the growth and development of this tissue, while some negatively related genes may have developed differences in function. In general, there were close correlations among those genes of the EXPA family that were highly expressed in fruit (Fig. 8b). At all stages of fruit development, the variation trends of EXPA subfamily expression were not exactly the same, and negative correlations of some genes were obvious (Fig. 8).

Five genes from the EXPA subfamily were identified to regulate fruit development by responding to Indole-3acetic acid (IAA) signals

Studies have shown that overexpression of AtEXPA10 in tobacco affects the size of reproductive organs, while overexpression of NtEXPA5 in tobacco increases the size of tobacco leaf and stem cells [49]. To further screen expansin proteins that potentially regulate fruit size, six genes with the highest homology to the previously 


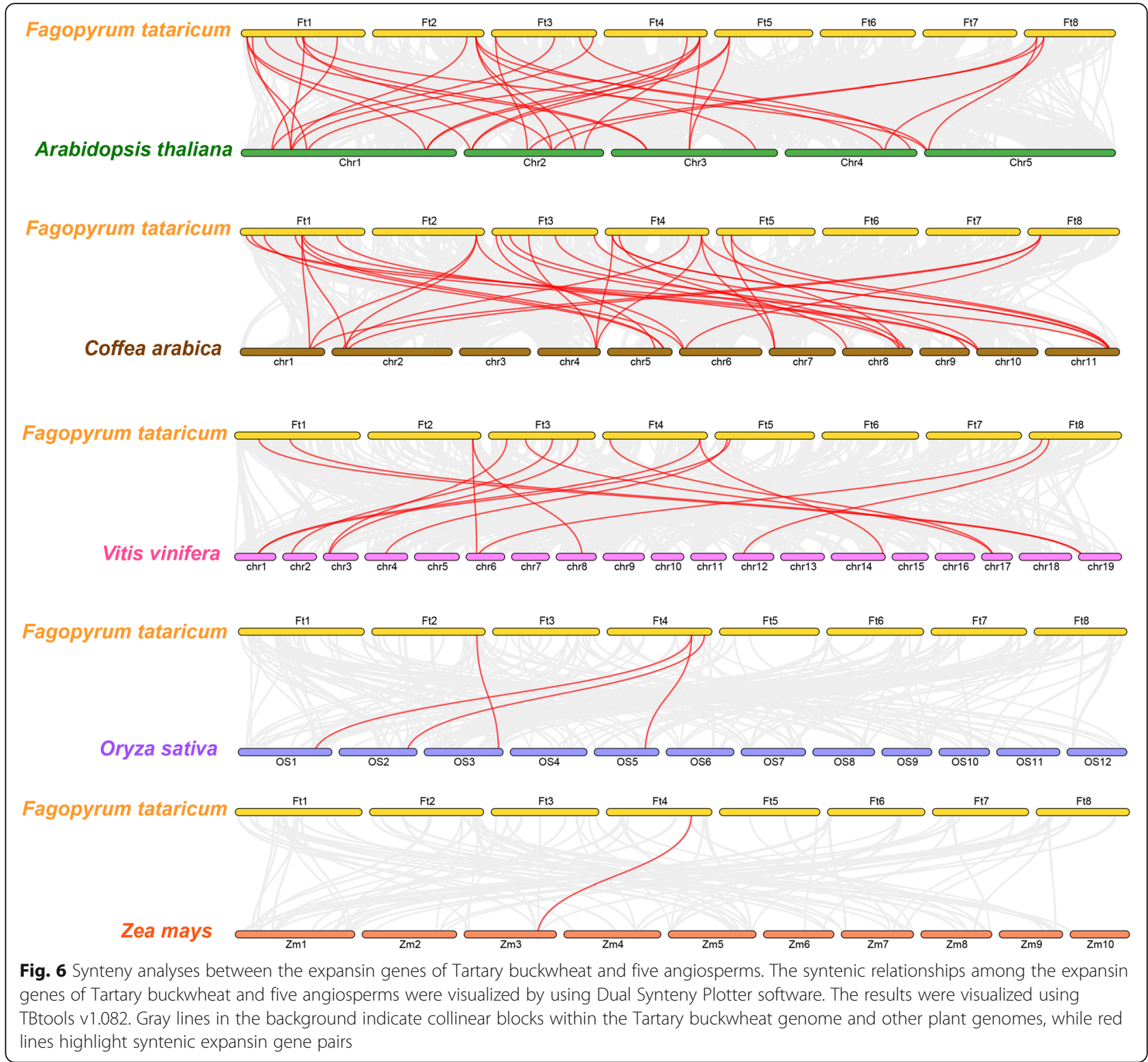

reported AtEXP10 (AT1G26770.2) were selected in the phylogenetic tree (Fig. 1). The expression of these six FtEXPA genes in BTB fruit and STB fruit was determined (Fig. 9a). The results showed that there were significant differences in the expression of the other five genes except FtPinG0006622100.01 in STB and BTB fruits. Among them, the expression of three genes (FtPinG0009591900.01, FtPinG0000209500.01 and FtPinG0004679600.01) in STB fruits was higher than that in BTB fruits, and the expression of the other two genes (FtPinG0006353400.01 and FtPinG0005157100.01) in BTB fruits was higher than that in STB fruits. Auxin plays an indispensable role in the expansion of plant organs [52]. The Tartary buckwheat fruit size reached a maximum at $19 \mathrm{DAP}$, and the auxin content in Tartary buckwheat fruits increased gradually from 13 DAP to 19 DAP [51]. FtPinG0006353400.01 and FtPinG000 5157100.01 were both highly expressed in BTB fruits, and their expression levels in the fruit development stage were consistent with the changes in the auxin content in fruits. Through the above results, we found genes that were differentially expressed in the STB and BTB fruits, which further narrowed the candidate range of fruit size-regulating genes.

The studies above showed that several EXPA subfamily genes (FtPinG0009591900.01, FtPinG0000209500.01, FtPinG0006353400.01, FtPinG0004679600.01 and FtPinG0005157100.01) were highly expressed in both the STB and BTB fruits, and there were significant differences in the expression of these genes between the 


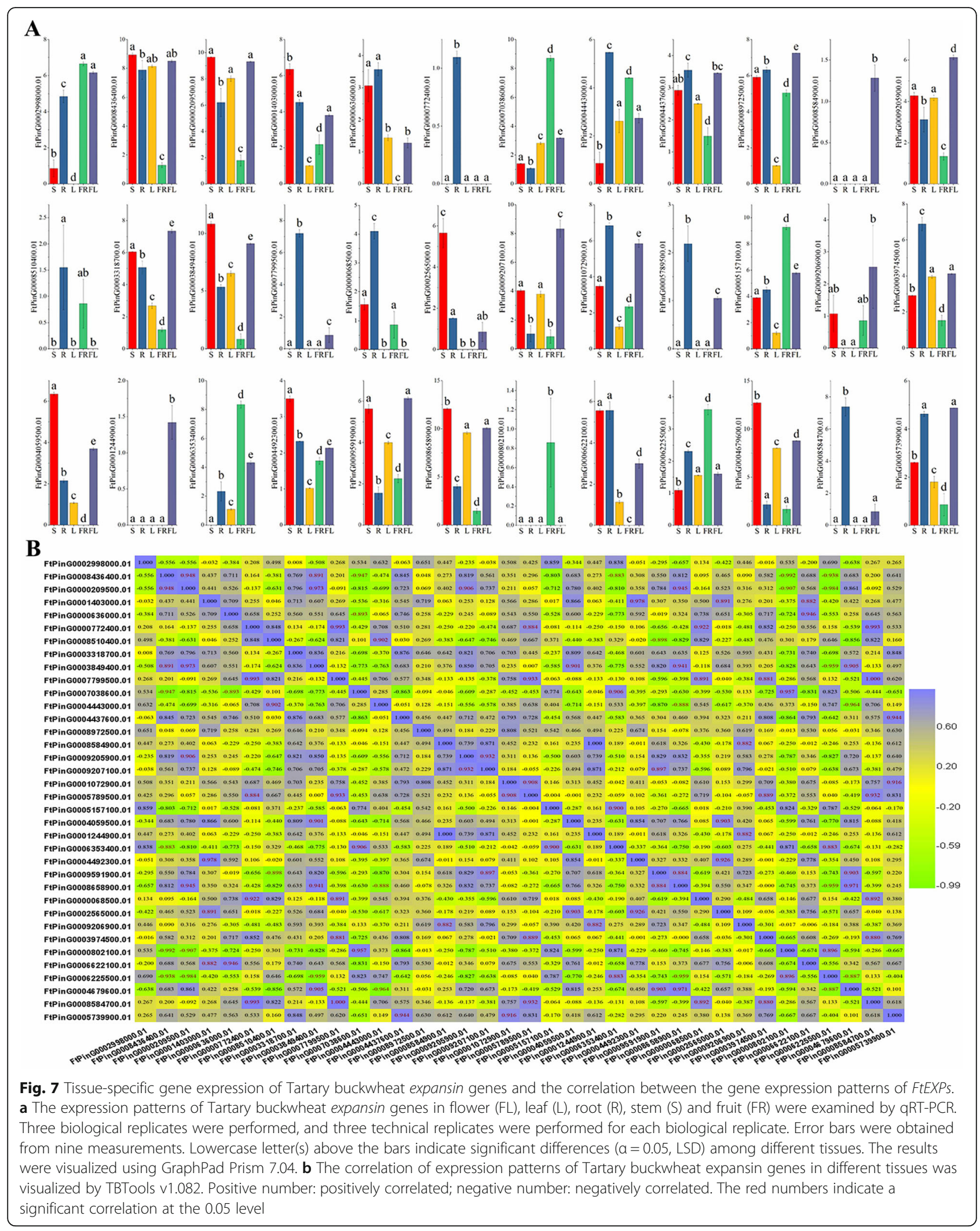




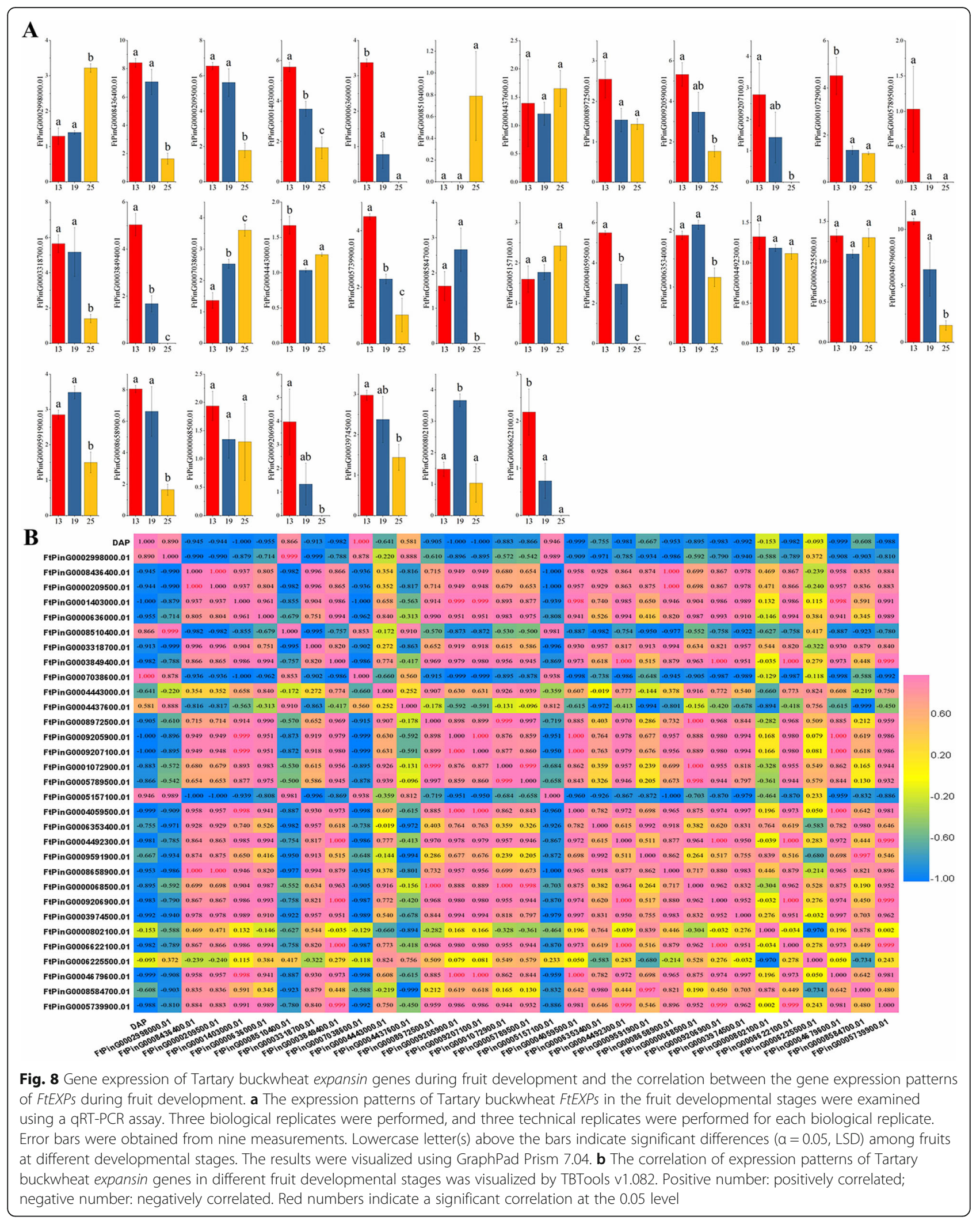



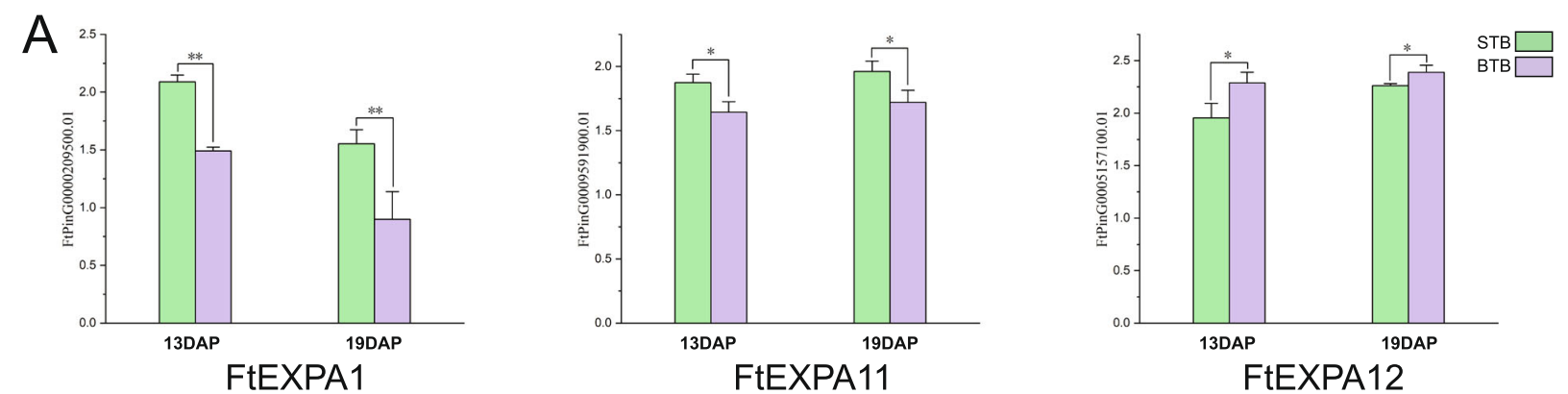

FtEXPA12
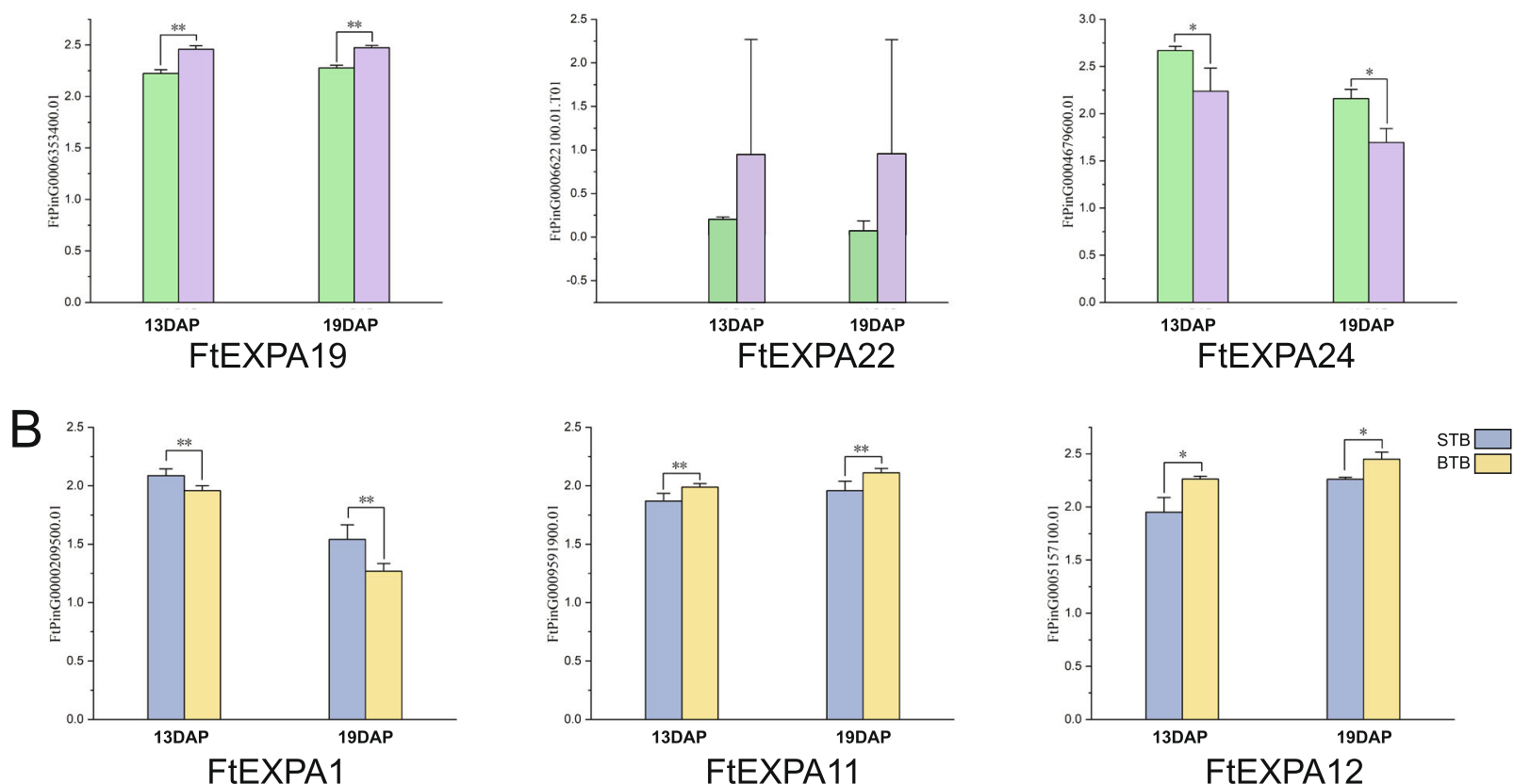

FtEXPA11

FtEXPA12
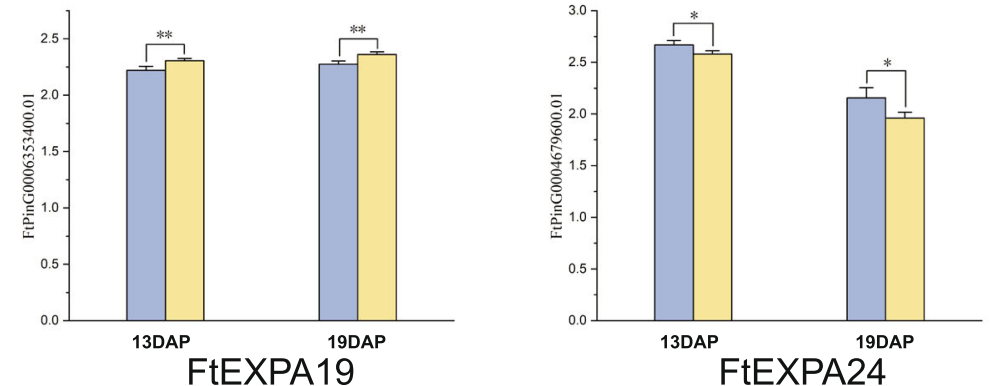

Fig. 9 Gene expression of six FtEXPA genes in the BTB and STB at 13 DAP and 19 DAP and the expression patterns of five FtEXPA genes from 13 DAP and 19 DAP of the STB with IAA treatment. a The expression patterns of six FtEXPA genes in BTB and STB at 13 DAP and 19 DAP were examined using a qRT-PCR assay. Three biological replicates were performed, and three technical replicates were performed for each biological replicate. Error bars were obtained from nine measurements. Asterisk above the bars indicate significant differences $\left({ }^{*} P<0.05 ;{ }^{* *} P<0.01\right)$ between the BTB and STB fruits. The results were visualized using GraphPad Prism 7.04. b The expression patterns of five FtEXPA genes in STB at 13 DAP and 19 DAP with IAA treatment were examined using a qRT-PCR assay. Three biological replicates were performed, and three technical replicates were performed for each biological repeat. Error bars were obtained from nine measurements. Asterisk above the bars indicate significant differences $\left({ }^{*} P<0.05 ;{ }^{* *} P<0.01\right)$ between mock and IAA. The results were visualized using GraphPad Prism 7.04 
two types of fruits. A previous study showed that the fruit weights of STB increased with exogenous auxin treatment [51]. Therefore, we measured the expression of these five genes in STB fruits under IAA treatment to further screen potential genes for regulating fruit size. The results showed that the expression of three genes (FtPinG0009591900.01, FtPinG0006353400.01 and FtPinG0005157100.01) increased and that the expression of two genes (FtPinG0004679600.01 and FtPinG00 00209500.01) decreased with IAA treatment (Fig. 9b).

\section{Discussion}

Expansin proteins can regulate many plant growth and development processes by participating in the synthetic modification of cell walls. As a result, the expansin gene family is valuable for plant growth and development [5]. In addition, expansin proteins are not found in animal and fungal species, while they are widespread in plants ranging from algae to higher plants, which also makes them of fascinating significance for studying the terrestrial evolution of plants. Based on our phylogenetic research, expansin proteins have at least one DPBB conserved domain in both algae and higher plants (Fig. S1). The EXPA subfamily has the largest number of members and was already present in algae. The EXPB subfamily originated later and had a smaller number of members. The surprising number of EXPA subfamily members in the basal terrestrial moss (M. polymorpha) may suggest that the family's genes expanded as plants transitioned to land (Fig. 1). Abundant gene duplication events drove the expansion of expansin families in angiosperms, and the $\mathrm{Ka} / \mathrm{Ks}$ values of most homologous EXP gene pairs in both monocotyledonous and dicotyledonous plants were all obviously less than 1 , which indicates that the expansin family received purification options after plant terrestrialization (Fig. 5).

Evolutionary analysis of expansin families provides valuable insight into the regulation of important agronomic traits in Tartary buckwheat genetics and breeding. Our first key finding is that the Tartary buckwheat EXPA subfamily expanded after gene duplication, and the evolution direction of the members varied. Compared with the other three subfamilies, the EXPA subfamily has the most members, as has been reported for other plants [46]. It has been reported that segmental duplication events contribute to gene expansion [53]. This conclusion is further supported by the finding that most members of the Tartary buckwheat EXPA subfamily undergo tandem and segmental duplication (Fig. 3, Fig. 5a). Studies have shown that gene duplication events can trigger family expansion [54], and the genes that undergo duplication have three evolutionary outcomes: maintaining the original conserved function, generating new functions or forming pseudogenes [55].
FtPinG0001244900.01-FtPinG0001244700.01 and FtPin G0009206900.01-FtPinG0009207100.01 are two pairs of tandem duplicated genes located on chromosomes 3 and 8 (Fig. 3), all of which are from the EXPA subfamily. Among them, the expression of FtPinG0009206900.01 and FtPinG0009207100.01 was obviously different (Fig. 8a), which indicates that they may have evolved into two genes with different functions. Furthermore, it was found that the motif compositions of these two genes were consistent, and it was suggested that the difference in function may originate from the difference in cisacting elements in the promoter region (Fig. 4, Fig. S3). In contrast, FtPinG0001244900.01 is only expressed in flowers, and FtPinG0001244700.01 had no expression (Fig. 8a), which indicated that FtPinG0001244700.01 may have become a pseudogene after duplication events. Whether pseudogenes are functional is an unresolved issue, and some argue that pseudogenes provide binding sites for transcription factors [56]. At least it is certain that these two pairs of tandem duplication genes from the same subfamily evolved in different directions after duplication occurred.

Another important finding is that duplication and loss of expansin proteins are common in representative angiosperms, and the conservative traits that were acquired from ancestors are favored by natural selection. This study found that two subfamilies (EXPA and EXPB) are conserved in representative angiosperms, which implies that they may already have existed in the common ancestor of angiosperms and were preserved after species differentiation. Studies have shown that certain plants that adapted to the land environment have lost some expansin proteins during evolution [57], which can provide a possible explanation for the larger number of EXLA subfamilies in algae, while few other species exist (Fig. 1). It has been reported that the expansion and contraction of gene families is a manifestation of the rapid adaptation of organisms to the environment [58]. However, new genes that formed with family expansion often faced harsh natural selection [59]. The $\mathrm{Ka} / \mathrm{Ks}$ values of FtEXPs were also all obviously less than 1 (Table S6), which indicated that the FtEXPs were purified during evolution. Specifically, although new genes were generated through evolutionary processes, the primitive functional genes that originated from ancestors were more conducive to adaptation to the environment [60]. Furthermore, EXPA subfamily genes may also have experienced motif loss and functional alternatives during evolution. Within the subfamily, motifs 5 and 10 do not usually coexist. One possible explanation is that the functions of motif 5 and motif 10 are redundant, and only one of them was preserved after a long period of evolution. Studies have also shown that the extra lost motif 1 (DPBB domain) is predicted to contain a signal 
peptide sequence (Fig. 4, Table S5) and can bind to cellulose [61], which may be a key factor for EXLA and EXLB not having cell wall-loosening functions.

Unfortunately, studies have shown that expansin proteins inevitably have negative effects on immune function while increasing fruit yield. Cell wall loosening is the direct cause of fruit organ enlargement [5], but expansion of the cell wall also leads to an increase in the gaps between cells, which may make plants vulnerable to external pathogens. Overexpression of indole-3-acetic acid-amido synthetase GH3-8 in rice induces IAA accumulation and then inhibits expansin protein expression [62]. Previous experimental evidence has confirmed that the disease resistance of rice was significantly enhanced, but its development was delayed [62]. This appears to indicate that we must make a choice between fruit enlargement and pathogen defense or other means to compensate for the lack of immune capacity that is caused by high expansin protein expression. Of the two FtEXPA subfamily genes (FtPinG0004679600.01 and FtPinG00 00209500.01) that were significantly downregulated after exogenous IAA treatment in our study (Fig. 9b), we suggest that the response of these two genes under IAA treatment may be similar to those mentioned in reports on rice [62]. For future studies, we should integrate other developmental, evolutionary, and ecological aspects to improve plant disease resistance to compensate for deficiencies in immune function after expansin protein expression.

\section{Conclusions}

Collectively, our research not only identified all expansin family members in the 12 representative plants during terrestrial processes, but also from the perspective of evolution, a blueprint was drawn for the selection and cultivation of the important agronomic traits of Tartary buckwheat fruit development. The expansin family originated from early algae that expanded rapidly after plant terrestrialization. EXPA subfamily members that are dependent on gene duplication expansion provide insights into Tartary buckwheat genetics and breeding. Notably, we identified five key candidate genes from the EXPA subfamily that could potentially regulate fruit size. Identification of target genes through evolutionary analyses at the whole-genome level can provide new insights for crop breeding. Our results will also contribute to improving the important agronomic properties of Tartary buckwheat. In addition, the research highlighted a new challenge regarding balancing the tradeoff between high yield and disease resistance of fruit, which provides an idea for future breeding.

\section{Methods}

Plant genome sequence acquisition and identification of the Expansin gene family

The Arabidopsis genome sequence was acquired from the TAIR database (https://www.arabidopsis.org/). The Tartary buckwheat genome was obtained from the Tartary buckwheat Genome Project (http://www.mbkbase. org/Pinku1) [40]. Other plant genome sequences $(M$. polymorpha, $V$. carteri, $C$. reinhardtii, $P$. patens, $S$. palustre, O. sativa, Z. mays, $V$. vinifera, $C$. arabica and A. trichopoda) were downloaded from the Phytozome database (http://www.phytozome.net/). The hidden Markov model (HMM) profiles of two domains (PF03330 and PF01357) were obtained from the Pfam protein family database (http://pfam.sanger.ac.uk/). HMMER3.0 was used to identify EXPs from the genomes. SMART [63], Pfam [64] and InterPro [65] were used to verify whether the identified genes had conserved domains and to remove the genes without conserved domains. Then, the expansin proteins identified above were BLASTp searched in NCBI to analyze whether they were part of the expansin family. We identified all expansin proteins from the genomes of 12 plants by using the above methods. Information on the isoelectric point (PI) and molecular weight $(\mathrm{Mw})$ was acquired from the ExPASy website (https://web.expasy.org/protparam/). The subcellular localizations of the FtEXP proteins were predicted with CELLO (http://cello.life.nctu.edu.tw/).

\section{Phylogenetic analyses, chromosomal location, intron- exon structure, motif composition and Cis-acting element analysis}

TBtools v1.082 was used to extract CDSs from all plant genomes and translate them into protein sequences [66]. The expansin protein sequences from different plants were aligned by using the Clustalx1.81 program [67]. The Clustalx1.81 parameters were defined as follows: in pairwise alignment, the gap opening penalty was 10 , and the gap extension penalty was 0.1 ; in multiple alignments, the gap opening penalty was also 10 , but the gap extension penalty was 0.2 . The phylogenetic tree of the expansin protein sequences in different plants was constructed with Mega 7.0 by the maximum likelihood method and 1000 bootstrap replications. The phylogenetic tree of Tartary buckwheat and A. thaliana was established to define the grouping of FtEXPs and was constructed by the above method. After that, the Clustalx1.81 program was used to align the expansin protein sequences of Tartary buckwheat and $A$. thaliana. Predictions of intron structures with expansin DNA sequences were performed by using the online Gene Structure Display Service 2.0 (http://gsds.gao-lab.org/). The conserved motifs of expansin proteins were determined by the MEME online program (http://meme-suite.org/tools/ 
meme), and the parameters were defined based on those used by Liu et al. [68]. The cis-acting elements that were 2000 bp upstream of all FtEXPs were predicted through the PlantCare online software (http://bioinformatics.psb. ugent.be/webtools/plantcare/html/).

\section{Chromosome distribution, duplication events, Syntenic analysis and $\mathrm{Ka} / \mathrm{Ks}$ ratio calculations of EXPs to homologous gene pairs in all angiosperms}

Gff files and sequencing files were used to obtain the chromosome localization information of FtEXPs. Analysis of FtEXP duplication events was performed through multiple collinear scanning toolkits (MCScanX) [69] and to visualize data through TBtools v1.082. The syntenic relationships between the expansin genes of Tartary buckwheat and five angiosperms were visualized by using Dual Synteny Plotter software and were visualized using TBtools v1.082. KaKs_Calculator 2.0 was used to calculate the synonymous (Ks) and nonsynonymous (Ka) substitutions of each homologous expansin gene pair and their ratios $(\mathrm{Ka} / \mathrm{Ks})[70]$.

\section{Plant growth}

The big fruit Tartary buckwheat accessions (BTB, XIQIAO) and small fruit Tartary buckwheat accessions (STB, MIQIAO) were cultivated at the experimental farm of the College of Life Sciences, Sichuan Agricultural University, China. We collected samples from three replicate plants. After 90 days of Tartary buckwheat germination, we collected flowers, stems, roots and leaves. We picked the fruits at 13, 19 and 25 days after pollination (DAP). All picked materials were rapidly placed in liquid nitrogen and were then kept at $-80^{\circ} \mathrm{C}$.

\section{Auxin treatment of STB}

In previous studies of the regulation of fruit size by FtARF2, STB was sprayed at the budding stage with 40, $70,100,130$, or $160 \mathrm{mg} \mathrm{L}^{-1}$ IAA. It was found that 100 $\mathrm{mg} \mathrm{L}^{-1}$ IAA was the best concentration for increasing fruit weight [68]. Therefore, STB plants were treated with $100 \mathrm{mg} \mathrm{L}^{-1}$ IAA in this study. After treatment, fruits at 13 DAP and 19 DAP were collected and placed at $-80^{\circ} \mathrm{C}$.

\section{Expression analysis of the FtEXPs}

The FtEXP expression in the stem, root, leaf, flower and fruit during different developmental stages (green fruit stage, 13 DAP; discoloration stage, 19 DAP; and initial maturity stage, 25 DAP) were measured by qRT-PCR. At the same time, the expression of FtEXPA genes in the BTB and STB fruits and in the STB fruits treated with IAA was also measured. The primers used in qRT-PCR (Table S8) were designed through the online software primer 3 (https://www.ncbi.nlm.nih.gov/tools/primer- blast/). The Tartary buckwheat histone $\mathrm{H} 3$ gene was used as an internal reference gene, and SYBR Premix Ex Taq II (TaKaRa) was used in qRT-PCR [71]. The correlative expression data were calculated using the $2^{-\Delta \Delta C T}$ method [72].

\section{Statistical analysis}

The obtained experimental data were processed and analyzed by GraphPad Prism 7.04, and the least significant difference test was used to compare the data.

\section{Data availability statement}

The datasets supporting the conclusions of this article are included in the article and its additional files.

\section{Abbreviations}

A. thaliana: Arabidopsis thaliana; BTB: Big fruit Tartary buckwheat; DAP: Days after pollination; EXP: Expansin; EXPA: a-expansin; EXPB: $\beta$-expansin; EXLA: Expansin-like A; EXLB: Expansin-like B; FtEXP: Fagopyrum tataricum expansin; FtEXPA: FtEXP in EXPA family; Gff: Generic feature format; GH45: Glycoside hydro-lase family-45; HMM: Hidden Markov Model; IAA: Indole-3-acetic acid; Mw: Molecular weight; PI: Isoelectric points; qRTPCR: Quantitative real-time polymerase chain reaction; STB: Small fruit Tartary buckwheat; TBGP: Tartary buckwheat genome project

\section{Supplementary Information}

The online version contains supplementary material available at https://doi. org/10.1186/s12864-021-07562-w.

\section{Additional file 1: Figure S1. Phylogenetic relationships and motif compositions of the expansin proteins from five different plant species. Outer layer: Phylogenetic trees were constructed using MEGA 7.0 with the maximum likelihood method. These phylogenetic trees were visualized by using the online tool Interactive Tree Of Life (iTOL) (http:// itol2.embl.de/). Inner layer: Distribution of the conserved motifs in expansin proteins. The conserved motifs of the expansin proteins were determined by the MEME online program (http://meme-suite.org/tools/ meme) and were visualized by TBtools v1.082. The differently colored boxes represent different motifs and their positions in each expansin protein sequence.}

Additional file 2: Figure S2. Protein motif model of the expansin protein family in representative species. (A) Motif model of the algal expansin protein family. The conserved motifs of the algal expansin proteins were determined by the MEME online program (http://memesuite.org/tools/meme) and were visualized by TBtools v1.082. (B) Motif model of the bryophyta expansin protein family. The conserved motifs of the bryophyta expansin proteins were determined by the MEME online program (http://meme-suite.org/tools/meme), and were visualized by TBtools v1.082. (C) Motif model of the monocotyledon expansin protein family. The conserved motifs of the monocotyledon expansin proteins were determined by the MEME online program (http://meme-suite.org/ tools/meme), and were visualized by TBtools v1.082. (D) Motif model of the dicotyledonous expansin protein family. The conserved motifs of dicotyledonous expansin proteins were determined by the MEME online program (http://meme-suite.org/tools/meme), and were visualized by TBtools v1.082

Additional file 3: Figure S3. Cis-acting element analysis of the expansin protein promoters from Tartary buckwheat. The cis-acting elements that were $2000 \mathrm{bp}$ upstream of all FtEXPs were predicted through the PlantCare online software (http://bioinformatics.psb.ugent.be/webtools/ plantcare/html/) and were visualized by TBtools v1.082. Blocks of different colors represent light responsiveness elements, low temperature responsiveness elements, salicylic acid responsiveness elements, abscisic acid responsiveness elements, MeJA responsiveness elements, auxin 
responsiveness elements, gibberellin responsiveness elements and defense and stress responsiveness elements.

Additional file 4: Table S1. Number of expansion family members in multiple species. Table $\mathbf{S 2}$ Gene ID of expansion in multiple species. Table S3 List of the 37 FtEXP genes identified in this study. Table S4 List of the Tartary buckwheat $37 \mathrm{FtEXP}$ genes identified in this study. Table S5 Analysis and distribution of conserved motifs in Tartary buckwheat expansin proteins. Table $\mathbf{S 6} \mathrm{Ka}$, Ks and $\mathrm{Ka} / \mathrm{Ks}$ value of synteny expansin gene pairs in angiosperms genome. Table $\mathbf{5 7}$ Synteny expansin gene pairs between Tartary buckwheat and other angiosperms. Table S8 Primer sequences for qRT-PCR.

\section{Acknowledgements}

We thank all the colleagues in our laboratory for providing useful discussions and technical assistance. We are very grateful to the editor and reviewers for critically evaluating the manuscript and providing constructive comments for its improvement.

\section{Authors' contributions}

M.-Y.L. planned and designed the research, and analyzed data. W.-J.S. and H.M.Y. wrote the original manuscript. W.-J.S. and M.-Y.L. determined the expression of genes by qRT-PCR. Z.-T.M. and H.C. identified FtEXPs and visualized their structures. H.-M.Y. and W.-J.S. performed the evolutionary analysis of FtEXPs and several different species. W.-J.S. and H.C. performed FtEXPS chromosome distribution, gene duplication and synteny analysis. H.C. reviewed and edited the manuscript. H.C. supervised the research. W.-J.S., H.M.Y. and M.Y.L. contributed equally. All authors read and approved the final manuscript.

\section{Funding}

This research was supported by function study of FtbHLH transcription factor regulating Tartary buckwheat fruit dehiscence (2021 YFH0086) of Sichuan province science and technology support program. Funds were used for the design of the study and collection, analysis, and interpretation of data and in writing the manuscript, as well as in the open access payment.

\section{Availability of data and materials}

The genome sequences of Tartary buckwheat used for identifying the FtEXPs in this study were located in the Tartary Buckwheat Genome Project (TBGP; http://www.mbkbase.org/Pinku1/). The Tartary buckwheat accessions (XIQIAO and MIQIAO) materials used in the experiment were supplied by Professor Wang Anhu of Xichang University. The datasets supporting the conclusions of this article are included with in the article and its Additional files.

\section{Declarations}

\section{Ethics approval and consent to participate}

These plant materials (XIQIAO and MIQIAO) are widely used all over the world and no permits are required for the collection of plant samples. The plant materials are maintained in accordance with the institutional guidelines of the College of Life Sciences, Sichuan Agricultural University, China. This article did not contain any studies with human participants or animals and did not involve any endangered or protected species.

\section{Consent for publication}

Not applicable.

\section{Competing interests}

The authors declare that they have no competing interests.

\section{Author details}

'College of Life Science, Sichuan Agricultural University, Ya'an 625014, China. ${ }^{2}$ Joint Center for Single Cell Biology, School of Agriculture and Biology, Shanghai Jiao Tong University, Shanghai 200240, China. ${ }^{3}$ State Key Laboratory of Crop Gene Exploration and Utilization in Southwest China, Key Laboratory of Major Crop Diseases and Rice Research Institute, Sichuan Agricultural University, Chengdu 611130, China.
Received: 22 September 2020 Accepted: 26 March 2021

Published online: 09 April 2021

\section{References}

1. Kenrick P, Crane P. The origin and early evolution of plants on land. Nature. 1997;389:33-9.

2. Wang S, Li L, Li H, Sahu SK, Wang H, Xu Y, et al. Genomes of early-diverging streptophyte algae shed light on plant terrestrialization. Nat Plants. 2020;6: 95-106.

3. Lamport D, Tan L, Held M, Kieliszewski M. The role of the primary Cell Wall in plant morphogenesis. Int J Mol Sci. 2018;19:2674.

4. Zhang B, Gao Y, Zhang L, Zhou Y. The plant Cell Wall: biosynthesis, construction, and functions. J Integr Plant Biol. 2020;63:251-72.

5. Marowa P, Ding A, Kong Y. Expansins: roles in plant growth and potential applications in crop improvement. Plant Cell Rep. 2016;35:949-65.

6. AbuQamar S. Expansins: Cell Wall remodeling proteins with a potential function in plant defense; 2018.

7. Wang DJ, Hu BZ. The role of expansin in plant growth and development. Plant Physiol. 2013;49:19-23.

8. Wang G, Gao Y, Wang J, Yang L, Song R, Li X, et al. Overexpression of two cambium-abundant Chinese fir (Cunninghamia lanceolata) a-expansin genes CIEXPA1 and CIEXPA2 affect growth and development in transgenic tobacco and increase the amount of cellulose in stem cell walls. Plant Biotechnol J. 2011;9(4):486-502.

9. Civello PM, Powell AL, Sabehat A, Bennett AB. An expansin gene expressed in ripening strawberry fruit. Plant Physiol. 1999;121(4):1273-80.

10. Muthusamy M, Kim JY, Yoon E, Kim J-A, Lee S. BrEXLB1, a Brassica rapa Expansin-like B1 gene is associated with root development, drought stress response, and seed germination. Genes. 2020;11:404.

11. Kuluev BR, Lebedev YP, Chemeris AV. Morphological and physiological characteristics of transgenic tobacco plants expressing expansin genes: AtEXP10 from Arabidopsis and PnEXPA1 from poplar. Russ J Plant Physiol. 2012;59(1):97-104.

12. He X, Zeng J, Cao F, Ahmed I, Zhang GP, Vincze E, et al. HvEXPB7, a novel $\beta$-expansin gene revealed by the root hair transcriptome of Tibetan wild barley, improves root hair growth under drought stress. J Exp Bot. 2015;66:erv436.

13. Muthusamy M, Kim J-A, Jeong M-J, Lee S. Blue and red light upregulate aexpansin 1 (EXPA1) in transgenic Brassica rapa and its overexpression promotes leaf and root growth in Arabidopsis. Plant Growth Regul. 2020;91: $75-87$.

14. Li Y, Jones L, McQueen-Mason S. Expansins and cell growth, vol. 6; 2004

15. Cosgrove D. Enzymes and other agents that enhance cell wall extensibility. Annu Rev Plant Physiol Plant Mol Biol. 1999;50:391-417.

16. Nikolaos G, Yennawar NH, Cosgrove DJ. Structural basis for entropy-driven cellulose binding by a type-a cellulose-binding module (CBM) and bacterial expansin. Proc Natl Acad Sci U S A. 2012;109(37):14830-5.

17. Sampedro J, Cosgrove D. The expansin superfamily. Genome Biol. 2005; $6: 242$

18. Valenzuela-Riffo F, Morales-Quintana L. Study of the structure and binding site features of FaEXPA2, an a-expansin protein involved in strawberry fruit softening. Comput Biol Chem. 2020:87:107279.

19. Valenzuela-Riffo F, Palma C, Ramos P, Morales-Quintana L. Molecular and structural insights into FaEXPA5, an alpha-expansin protein related with cell wall disassembly during ripening of strawberry fruit. Plant Physiol Biochem. 2020:154:581-9.

20. Kende H, Bradford K, Brummell D, Cho H-T, Cosgrove D, Fleming A, et al. Nomenclature for members of the expansin superfamily of genes and proteins. Plant Mol Biol. 2004;55:311-4.

21. Cosgrove D, Bedinger P, Durachko DM. Group I allergens of grass pollen as Cell Wall-loosening agents. Proc Natl Acad Sci U S A. 1997;94:6559-64.

22. Yi L, Choi D, Kende H. Expansins: ever-expanding numbers and functions. Curr Opin Plant Biol. 2001;4(6):527-32

23. Sarma Bordoloi K, Dihingia P, B Krishnatreya D, Agarwala N. Genome-wide identification, characterization and expression analysis of the expansin gene family under drought stress in tea (Camellia sinensis L.). Plant Sci Today. 2021:8:32-44.

24. Lu Y, Liu L, Wang X, Han Z, Ouyang B, Zhang J, et al. Genome-wide identification and expression analysis of the expansin gene family in tomato. Mol Genet Genom Mgg. 2016;291(2):597. 
25. Zhang W, Yan H, Chen W, Liu J, Jiang C, Jiang H, et al. Genome-wide identification and characterization of maize expansin genes expressed in endosperm, vol. 289; 2014.

26. Zhu Y, Wu N, Song W, Yin G, Qin Y, Yan Y, et al. Soybean (Glycine max) expansin gene superfamily origins: segmental and tandem duplication events followed by divergent selection among subfamilies. BMC Plant Biol. 2014;14(1):93.

27. Lv L-M, Zuo D-Y, Wang X-F, Cheng H-L, Zhang Y-P, Wang Q-L, et al. Genome-wide identification of the expansin gene family reveals that expansin genes are involved in fibre cell growth in cotton. BMC Plant Biol. 2020;20:223-36.

28. Chen S, Ren H, Luo Y, Feng C, Li H. Genome-wide identification of wheat (Triticum aestivum L.) expansin genes and functional characterization of TaEXPB1A. Environ Exp Bot. 2021;182:104307.

29. Cosgrove DJ. Loosening of plant cell walls by expansins. Nature. 2000; 407(6802):321-6.

30. Lin C, Choi HS, Cho HT. Root hair-specific EXPANSIN A7 is required for root hair elongation in Arabidopsis. Mol Cell. 2011;31(4):393.

31. An Y, Minjie W, Limei $Y$, Rui H, Imran A, Yinbo G. AtEXP2 is involved in seed germination and abiotic stress response in Arabidopsis. PLoS One. 2014;9(1): e85208.

32. Javier S, Mara G, Lian-Chao L, Cosgrove DJ. Evolutionary divergence of $\beta$ expansin structure and function in grasses parallels emergence of distinctive primary cell wall traits. Plant J. 2015;81(1):108-20.

33. Cosgrove DJ. Plant expansins: diversity and interactions with plant cell walls. Curr Opin Plant Biol. 2015;25:162-72.

34. Quinet M, Angosto T, Yuste-Lisbona F, Blanchard-Gros R, Bigot S, Martínez J, et al. Tomato fruit development and metabolism. Front Plant Sci. 2019;10:1554.

35. Shinozaki Y, Nicolas P, Fernandez Pozo N, Ma Q, Evanich D, Shi Y, et al. High-resolution spatiotemporal transcriptome mapping of tomato fruit development and ripening. Nat Commun. 2018;9:364.

36. Joshi DC, Chaudhari GV, Sood S, Kant L, Pattanayak A, Zhang K, et al. Revisiting the versatile buckwheat: reinvigorating genetic gains through integrated breeding and genomics approach. Planta. 2019;250:783-801.

37. Zhu F. Chemical composition and health effects of Tartary buckwheat. Food Chem. 2016;203:231-45.

38. Yiming Z, Hong W, Linlin C, Xiaoli Z, Wen T, Xinli S. Evolution of nutrient ingredients in tartary buckwheat seeds during germination. Food Chem. 2015;186:244-8.

39. Wójcicki J, Wiszniewska B, Samochowiec L, Rózewicka L. Extractum Fagopyri reduces atherosclerosis in high-fat diet fed rabbits. Die Pharmazie. 1995:50:560-2.

40. Zhang L, Li X, Ma B, Gao Q, Du H, Han Y, et al. The Tartary buckwheat genome provides insights into Rutin biosynthesis and abiotic stress tolerance. Mol Plant. 2017;10(9):1224-37.

41. Araújo S, Beebe S, Crespi M, Bruno D, González E, Gruber V, et al. Abiotic stress responses in legumes: strategies used to cope with environmental challenges. Crit Rev Plant Sci. 2015;34:237-80.

42. War AR, Sharma HC. Effect of jasmonic acid and salicylic acid induced resistance in groundnut on Helicoverpa armigera. Physiol Entomol. 2014;39:136-42.

43. Sharma M, Gupta S, Majumder B, Maurya V, Deeba F, Alam A, et al. Salicylic acid mediated growth, physiological and proteomic responses in two wheat varieties under drought stress. J Proteome. 2017;163:28-51.

44. $\mathrm{Xu} \mathrm{J-H}$, Messing J. Diverged copies of the seed regulatory Opaque-2 gene by a segmental duplication in the progenitor genome of Rice, sorghum, and maize. Mol Plant. 2008;1:760-9.

45. Kuluev B, Knyazev A, Lebedev Y, Chemeris A. Morphological and physiological characteristics of transgenic tobacco plants expressing expansin genes: AtEXP10 from Arabidopsis and PnEXPA1 from poplar. Russ J Plant Physiol. 2011;59:97-104

46. Han Z, Yanlin L, Deng X, Liu D, Liu Y, Hu Y, et al. Genome-wide identification and expression analysis of expansin gene family in common wheat (Triticum aestivum L.). BMC Genomics. 2019:20:101-20.

47. Shin J-H, Jeong D-H, Park M, An G. Characterization and transcriptional expression of the alpha-expansin gene family in rice. Mol Cell. 2005;20:210-8.

48. Rose J, Lee H, Bennett A. Expression of a divergent Expansin gene is fruitspecific and ripening-regulated. Proc Natl Acad Sci U S A. 1997;94:5955-60.

49. Kuluev B, Safiullina M, Knyazev A, Chemeris A. Effect of ectopic expression of NtEXPA5 gene on cell size and growth of organs of transgenic tobacco plants. Russ J Dev Biol. 2013;44:34-41.
50. Heng W, Yang J, Hou Z, Li F, Jia B, Liu P, et al. Characterization and expression analysis of PbEXP genes in the epidermis of pear (Pyrus bretschneideri Rehd.). Plant Growth Regul. 2017;84:1-9.

51. Liu M, Ma Z, Zheng T, Sun W, Zhang Y, Jin W, et al. Insights into the correlation between physiological changes in and seed development of tartary buckwheat (Fagopyrum tataricum Gaertn.). BMC Genomics. 2018; 19(1):648.

52. Perrot-Rechenmann C. Cellular responses to Auxin: division versus expansion. Cold Spring Harb Perspect Biol. 2010;2(5):a001446.

53. Dossa K, Diouf D, Cisse N. Genome-wide investigation of Hsf genes in sesame reveals their segmental duplication expansion and their active role in drought stress response. Front Plant Sci. 2016;7:1522.

54. Qiao X, Li Q, Yin H, Qi K, Li L, Wang R, et al. Gene duplication and evolution in recurring polyploidization-diploidization cycles in plants. Genome Biol. 2019;20(1):38-61.

55. Nan Q, Qian D, Niu Y, He Y, Tong S, Niu Z, et al. Plant actin-depolymerizing factors possess opposing biochemical properties arising from key amino acid changes throughout evolution. Plant Cell. 2017;29(2):395-408.

56. Jianbo X, Ying L, Xiaomin L, Yiyang Z, Bailian P. Evolutionary origins of Pseudogenes and their association with regulatory sequences in plants. Plant Cell. 2019;31:563-78.

57. Hepler NK, Bowman A, Carey RE, Cosgrove DJ. Expansin gene loss is a common occurrence during adaptation to an aquatic environment. Plant J. 2019;101:66680.

58. Schilling S, Kennedy A, Pan S, Jermiin L, Melzer R. Genome-wide analysis of MIKC -type MADS -box genes in wheat: pervasive duplications, functional conservation and putative neofunctionalization. New Phytol. 2019;225:51129.

59. Theißen G, Rümpler F, Gramzow L. Array of MADS-box genes: facilitator for rapid adaptation? Trends Plant Sci. 2018;23:563-76.

60. Hurst LD. The Ka/Ks ratio: diagnosing the form of sequence evolution. Trends Genet. 2002;18(9):486.

61. Gray-Mitsumune M, Mellerowicz E, Schrader J, Winzéll A, Sterky F, Blomqvist $K$, et al. Expansins abundant in secondary xylem belong to subgroup a of the -Expansin gene family. Plant Physiol. 2004;135:1552-64

62. Ding X, Cao Y, Huang L, Zhao J, Xu C, Li X, et al. Activation of the Indole-3acetic acid-Amido Synthetase GH3-8 suppresses Expansin expression and promotes salicylate- and Jasmonate-independent basal immunity in Rice. Plant Cell. 2008;20(1):228-40.

63. Ivica $L$, Tobias $D$, Peer B. SMART 7: recent updates to the protein domain annotation resource. Nucleic Acids Res. 2011:D1:D1.

64. Finn RD, Bateman A, Clements J, Coggill P, Eberhardt RY, Eddy SR, et al. Pfam: the protein families database. Nucleic Acids Res. 2013;42(D1):D222-30.

65. Zdobnov EM, Rolf A. InterProScan - an integration platform for the signature-recognition methods in InterPro. Bioinformatics. 2001;9:9.

66. Chen $\mathrm{C}$, Chen $\mathrm{H}$, Zhang Y, Thomas H, Frank M, He Y, et al. TBtools: An integrative toolkit developed for interactive analyses of big biological data. Mol Plant. 2020:13:1194-202.

67. Thompson JD, Gibson TJ, Plewniak F, Jeanmougin F, Higgins DG. The ClustalX windows interface: flexible strategies for multiple sequence alignment aided by quality analysis tools. Nucleic Acids Res. 1997;25(25): 4876-82.

68. Liu M, Ma Z, Wang A, Zheng T, Huang L, Sun W, et al. Genome-wide investigation of the Auxin response factor gene family in Tartary buckwheat (Fagopyrum tataricum). Int J Mol Sci. 2018;19(11):3526-44.

69. Wang Y, Tang H, Debarry J, Tan X, Li J, Wang X, et al. MCScanX: a toolkit for detection and evolutionary analysis of gene synteny and collinearity. Nucleic Acids Res. 2012;40:e49.

70. Wang D, Zhang Y, Zhang Z, Zhu J, Yu J. KaKs_Calculator 2.0:a toolkit incorporating gamma-series methods and sliding window strategies. Genom Proteomics Bioinform. 2010;8(1):77-80

71. Liu M, Huang L, Ma Z, Sun W, Wu Q, Tang Z, et al. Genome-wide identification, expression analysis and functional study of the GRAS gene family in Tartary buckwheat (Fagopyrum tataricum). BMC Plant Biol. 2019;19(1):342-59.

72. Livak KJ, Schmittgen TD. Analysis of relative gene expression data using real-time quantitative PCR and the 2(-Delta Delta $C(T))$ method. Methods. 2012;25(4):402-8.

\section{Publisher's Note}

Springer Nature remains neutral with regard to jurisdictional claims in published maps and institutional affiliations. 